OPEN ACCESS

Edited by:

Tetsuro Miyazaki,

Juntendo University Urayasu

Hospital, Japan

Reviewed by:

Tomotaka Dohi,

Juntendo University, Japan

Shohei Ouchi,

Juntendo University, Japan

${ }^{*}$ Correspondence:

Yingxin Zhao

zyingxinmi@163.com

Jianwei Zhang

zhangjianwei_2003@sina.com

Specialty section: This article was submitted to Atherosclerosis and Vascular

Medicine,

a section of the journal

Frontiers in Cardiovascular Medicine

Received: 03 December 2020 Accepted: 08 January 2021

Published: 19 February 2021

Citation:

Hu C, Liu J, Han H, Sun Y, Cheng Y,

Liu Y, Gao A, Zhou Y, Zhang J and Zhao Y (2021) A Synergistic Effect of $L p(a)$ and GRACE Score on

Cardiovascular Risk in Acute Coronary

Syndrome Patients Undergoing Percutaneous Coronary Intervention:

A Cohort Study From China.

Front. Cardiovasc. Med. 8:637366.

doi: 10.3389/fcrm.2021.637366

\section{A Synergistic Effect of Lp(a) and GRACE Score on Cardiovascular Risk in Acute Coronary Syndrome Patients Undergoing Percutaneous Coronary Intervention: A Cohort Study From China}

\author{
Chengping Hu, Jinxing Liu, Hongya Han, Yan Sun, Yujing Cheng, Yan Liu, Ang Gao, \\ Yujie Zhou, Jianwei Zhang* and Yingxin Zhao*
}

\author{
Department of Cardiology, Beijing Institute of Heart Lung and Blood Vessel Disease, Beijing Anzhen Hospital, Capital Medical \\ University, Beijing, China
}

Objectives: Lipoprotein(a) $[\mathrm{Lp}(\mathrm{a})]$ has been thought as an independent risk factor for atherosclerotic cardiovascular disease (ASCVD). The Global Registry of Acute Coronary Events (GRACE) score is used to predict the risk of death or death/non-fatal myocardial infarction in patients with acute coronary syndromes (ACS). It suggests that there may be a synergism between Lp(a) and the GRACE risk score on predicting cardiovascular events. Accordingly, this study aimed to test the hypothesis that Lp(a)-related cardiovascular risk could be significantly modulated by the GRACE risk score in patients with ACS undergoing percutaneous coronary intervention (PCI).

Methods: Patients hospitalized with ACS undergoing PCI were enrolled and followed up for 18 months. The primary outcome was the composite of death, non-fatal myocardial infarction, non-fatal stroke, and unplanned repeat revascularization. A Cox proportional hazard regression model was used to determine the relationship between $L p(a)$ and cardiovascular events.

Results: A total of 6,309 patients were included (age: $60.1 \pm 10.06$ years, male: $75.2 \%$, BMl: $\left.26.2 \pm 10.57 \mathrm{~kg} / \mathrm{m}^{2}\right)$. A total of $310(4.9 \%)$ cardiovascular events occurred. When the overall population was stratified by a GRACE score of 91 or less vs. more than 91 and by tertiles of Lp(a), higher Lp(a) was significantly associated with cardiovascular events only when the GRACE score was <91(tertile 2 vs. tertile 1: $\mathrm{HR} 1.31,95 \% \mathrm{Cl}: 0.86-1.98, P=0.205$; tertile 3 vs. tertile 1: HR 1.94, 95\% Cl: 1.32-2.84, $P=0.001 ; P=0.002)$. However, no such significant correlation between cardiovascular events and Lp(a) emerged in the case of a GRACE score 91 or less, and there was a significant interaction for cardiovascular events between $\operatorname{Lp}(\mathrm{a})$ tertiles and dichotomized GRACE scores $(P<0.001)$. 
Conclusions: In ACS patients undergoing $\mathrm{PCl}$, there was a synergistic effect between the GRACE risk score and on-statins $L p(a)$ on predicting cardiovascular events. This finding could help us more accurately identify patients who would benefit most from Lp(a)-lowering treatment.

Keywords: lipoprotein(a), acute coronary syndrome, percutaneous coronary intervention, GRACE score, prognosis

\section{INTRODUCTION}

Despite effective low-density lipoprotein cholesterol (LDL-C)lowering treatment, a significant residual risk remains. In IMPROVE-IT (Improved Reduction of Outcomes: Vytorin Efficacy International Trial), the rate of cardiovascular risk remained $32.7 \%$ even after LDL-C reached $54 \mathrm{mg} / \mathrm{dl}$ or less, suggesting that LDL-C-lowering treatment might not optimally reduce cardiovascular risk (1). Lipoprotein(a) $[\mathrm{Lp}(\mathrm{a})]$ has been considered an independent risk factor for atherosclerotic cardiovascular disease (ASCVD), and high $\mathrm{Lp}(\mathrm{a})$ remains a risk factor despite LDL-C being $<70 \mathrm{mg} / \mathrm{dl}(2-4)$. The JUPITER trial (Justification for the Use of Statins in Prevention: An Intervention Trial Evaluating Rosuvastatin) enrolled 9612 patients and indicated that higher on-statin Lp(a) was associated with greater cardiovascular risk independent of low-density lipoprotein cholesterol (LDL-C) [adjusted hazard ratio (HR) 1.27; 95\% confidence interval (CI), 1.01-1.59, $P=0.04]$ (2). Thus, $\mathrm{Lp}$ (a) was considered a risk factor and potential therapeutic target (5-7).

Evidence has suggested that Lp(a) could be reduced effectively by $\sim 20-40 \%$ for proprotein convertase subtilisin/kexin type 9 (PCSK9) inhibitors, cholesteryl ester transfer protein inhibitors, and mipomersen and by $70-90 \%$ with antisense oligonucleotides (8). Despite recent progress, which patients are likely to benefit the most from a reduction in $\mathrm{Lp}(\mathrm{a})$ remains uncertain.

$\mathrm{Lp}(\mathrm{a})$ is believed to have pro-thrombotic properties, suggesting that there might be a synergistic effect of $L p(a)$ and systemic thrombotic risk on cardiovascular events. The Global Registry of Acute Coronary Events (GRACE) score has a very good discriminative ability for predicting death in patients with ACS regardless of ST-segment elevation myocardial infarction (STEMI), non-ST-segment elevation myocardial infarction (NSTEMI), or unstable angina (9). The National Institute for Health and Care Excellence (NICE) independently tested all of the risk scores (GRACE, TIMI, PURSUIT, PREDICT, EMMACE, SRI, AMIS, UA risk score) in 64,312 patients and demonstrated that the GRACE score performs significantly better than other risk scores with a c statistic of 0.825 (95\% CI 0.82-0.83) (10). In addition, current guidelines also recommend the GRACE score to calculate patients' short-term and long-term risks of fatal or non-fatal cardiovascular events (11). Therefore, we employ the GRACE post-discharge risk score to assess the risk of cardiovascular events in this study.

Accordingly, we tested the hypothesis that $\mathrm{Lp}(\mathrm{a})$-related cardiovascular risk could be significantly modulated by the GRACE score in patients with acute coronary syndromes (ACS) undergoing percutaneous coronary intervention (PCI), and it could better identify patients who would benefit most from Lp(a) lowering.

\section{METHODS}

\section{Study Design and Patients}

From January 2018 to December 2018, ACS patients hospitalized for PCI were enrolled in this study. The key exclusion criteria were a body mass index $(\mathrm{BMI})>45 \mathrm{~kg} / \mathrm{m}^{2}$, suspected familial hypertriglyceridemia (triglyceride $\geq 5.65 \mathrm{mmol} / \mathrm{L}$ ), severe hepatic and renal insufficiency (eGFR $<30 \mathrm{ml} / \mathrm{min}$ ), left ventricular ejection fraction (LVEF) $<30 \%$, and use of fibrate and PCSK9 inhibitors which have great effects on $\operatorname{Lp}(\mathrm{a})$ and malignancy. Moreover, patients with incomplete key variables including GRACE score variables and Lp(a) were also excluded. The institutional review board of Beijing Anzhen Hospital, Capital Medical University, approved the study protocol. A waiver of informed consent was granted, and patients' personal information was concealed.

\section{Measurements}

Patients fasted for at least $8 \mathrm{~h}$ before blood draw. Blood was drawn on the day of admission for fasting patients, and the next morning for non-fasting patients. Moreover, measurement was performed on the day of drawing blood. For patients with STEMI, blood samples were collected immediately on admission. Lipid profiles were measured on the same day of collection. Lipid parameters, glycosylated hemoglobin (HbAlc), and fasting plasma glucose (FPG) were quantified by clinical standard laboratory techniques. ELISA (Biocheck Laboratories, Toledo, $\mathrm{OH}$, USA) was performed to detect $\mathrm{Lp}(\mathrm{a})$ and high-sensitivity C-reactive protein (hs-CRP) levels. In addition, the GRACE postdischarge score with eight variables was calculated to estimate the risk of cardiovascular events (12).

\section{Treatment and Procedures}

Aspirin and clopidogrel or ticagrelor was given preoperatively and unfractionated heparin (70-100 IU/kg) intraoperatively. All of the patients took statins with or without ezetimibe unless serious complications occurred. Coronary intervention was performed using 6 or $7 \mathrm{~F}$ guiding catheters through a radial approach. Balloon pre-dilation was followed by secondgeneration drug-eluting stents. The type of stent, optical coherence tomography (OCT), intravascular ultrasound (IVUS), and fractional flow reserve (FFR) were left to the discretion of the interventionalists. All medications and operations were performed in compliance with current guidelines (13). 
TABLE 1 | Baseline characteristics of patients according to GRACE score.

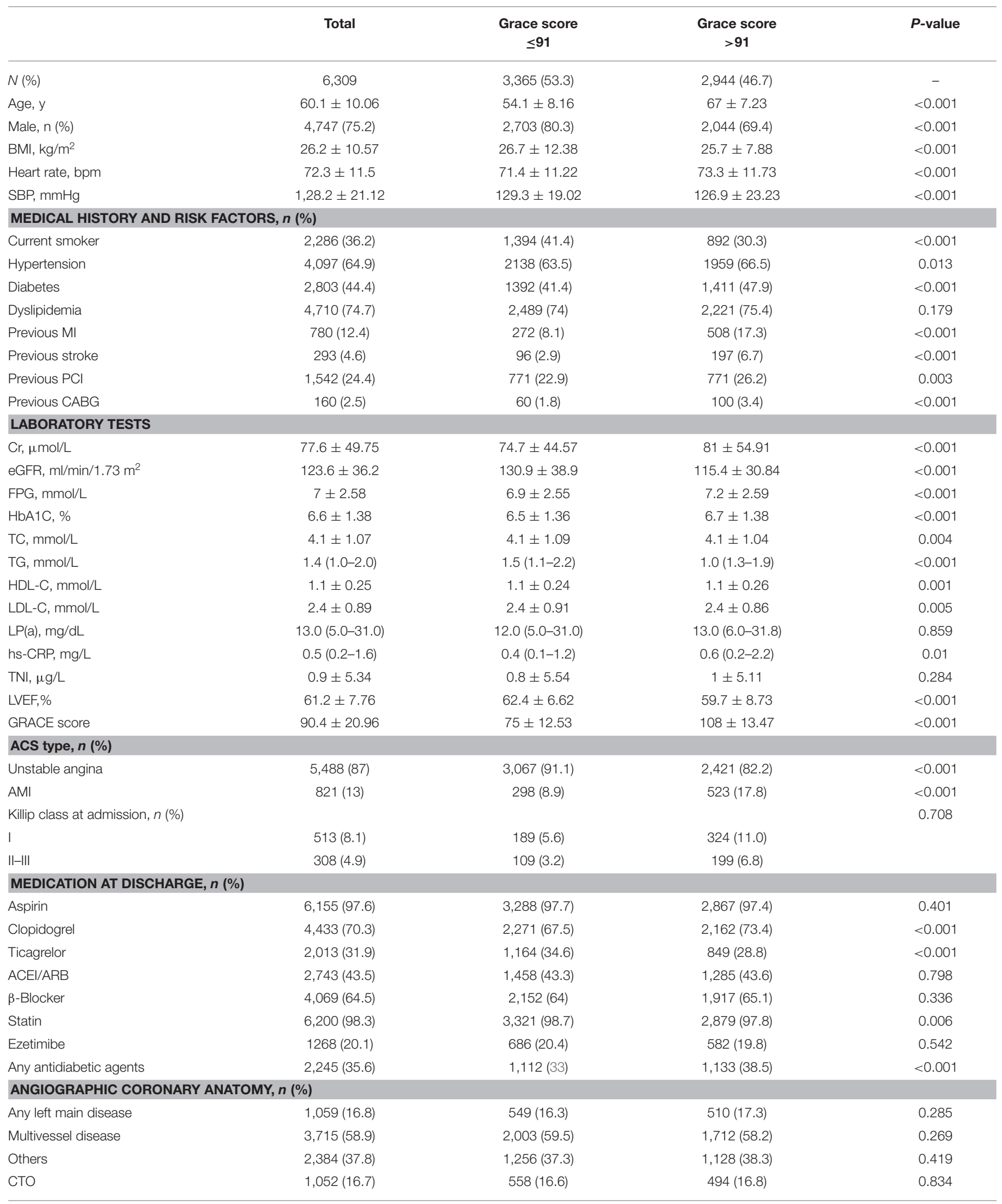


TABLE 1 | Continued

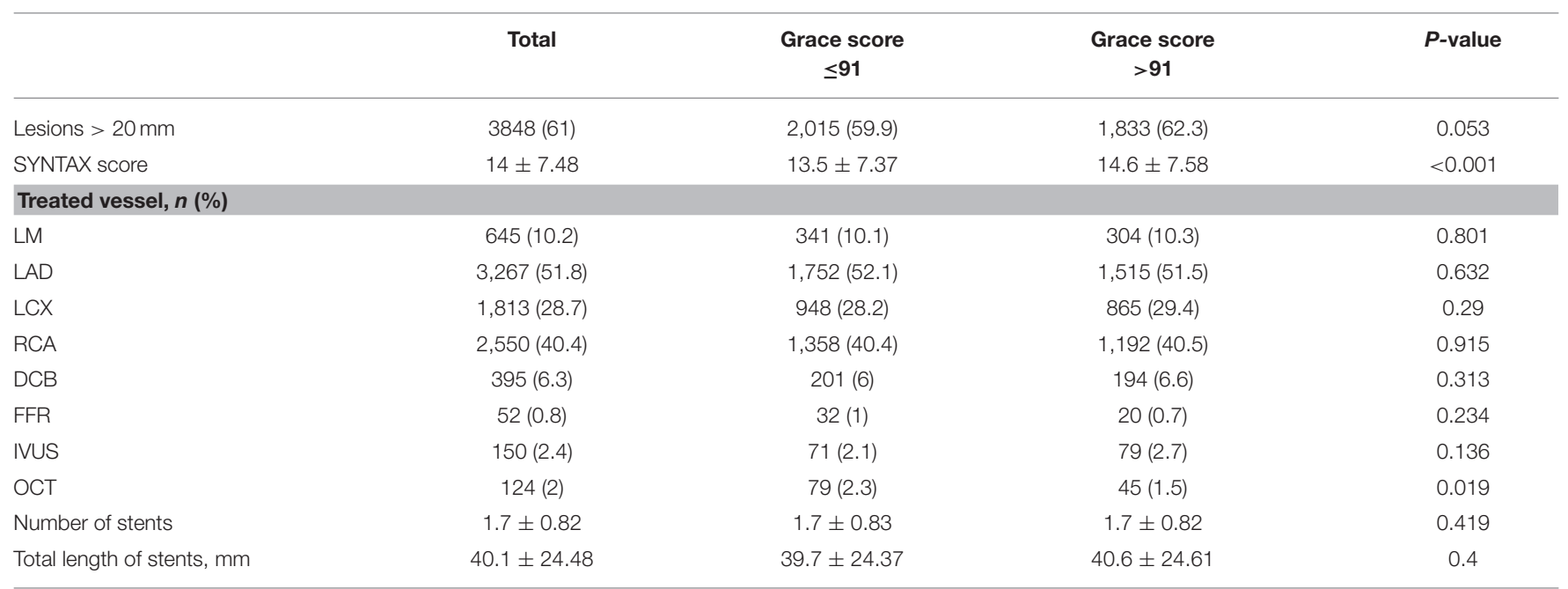

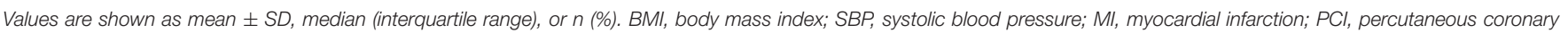

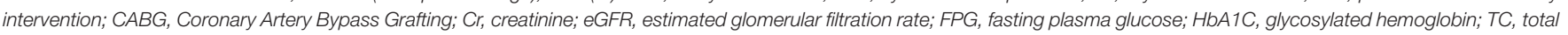

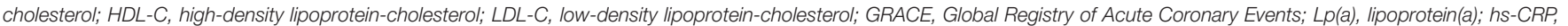

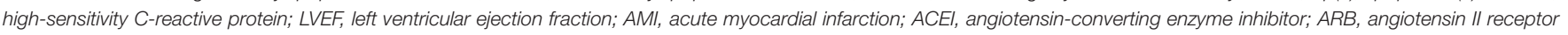

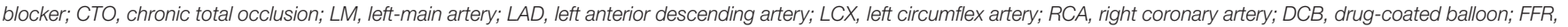
fractional flow reserve; IVUS, intravascular ultrasound; OCT, optical coherence tomography.

\section{Outcomes}

Follow-up for 18 months was performed by telephone conversation, and the time to index event was used for analysis. The hospital records were also provided data for screening clinical events. The primary outcome for the current analysis was a composite of all-cause death, non-fatal myocardial infarction (MI), non-fatal stroke, or unplanned repeat revascularization. Death was defined as any death resulting from any cause. Incident stroke was defined as acute cerebral infarction according to the typical symptoms or imaging (14). Incident MI was defined on the basis of the fourth universal definition of myocardial infarction (15). Unplanned revascularization was defined as any unexpected PCI or surgical bypass after the index procedure on either target or non-target vessel $(14,16)$. Unstable angina was defined as acute chest pain with or without electrocardiographic abnormalities and normal cardiac enzymes (17). Dyslipidemia was defined as self-reported use of any lipid-lowering drug, fasting TG $>150 \mathrm{mg} / \mathrm{dL}, \mathrm{HDL}-\mathrm{C}<40 \mathrm{mg} / \mathrm{dL}$, and/or LDL-C $>130 \mathrm{mg} / \mathrm{dL}$. Diabetes was defined as taking hypoglycemic agents, a fasting plasma glucose of $\geq 7.0 \mathrm{mmol} / \mathrm{L}$, non-fasting plasma glucose of $\geq 11.10 \mathrm{mmol} / \mathrm{L}$, or self-reported disease (18). Hypertension was defined as use of anti-hypertensive drugs, systolic blood pressure $\geq 140 \mathrm{~mm} \mathrm{Hg}$, or diastolic blood pressure $\geq 90 \mathrm{mmHg}(19)$.

\section{Statistical Analysis}

The baseline characteristics are presented according to the median baseline GRACE score. Continuous variables are presented as the mean \pm standard deviation (SD) or median (interquartile range) and were compared with the $t$ test or the Mann-Whitney $U$ test. Categorical variables are reported as numbers (percentage) and compared using the $\chi^{2}$ test (Fisher's exact test). Cox proportional hazard regression models were used to determine the relationship between cardiovascular events and $\mathrm{Lp}$ (a) with the backward stepwise method as a variable selection method, and the model was conducted by fully adjusting for variables, including age, sex, BMI, smoking status, hypertension, previous MI, previous stroke, SYNTAX score, number of stents, total length of stents, high sensitivity C-reactive protein (hsCRP), HDL-C, LDL-C, and lipid-lowering medication use. The statistical interaction between $\mathrm{Lp}(\mathrm{a})$ and GRACE score was examined by incorporating multiplicative interaction terms in the same model. The Kaplan-Meier curves were plotted to illustrate the cumulative incidence of cardiovascular events over time, and they were compared by the log-rank test. Sensitivity analyses were performed in patients with or without diabetes. Moreover, we provide the comparisons of baseline characteristics between participants who were included in the final analyses or not to test whether the lost data were random. Statistical analyses were performed using SPSS software, version 24.0 (IBM Corp., Armonk, NY, USA). Statistical significance was considered as $P<0.05$ (two-tailed).

\section{RESULTS}

A total of 9,285 patients met the inclusion criteria, with 2,976 excluded due to loss to follow-up $(n=698)$ or the exclusion criteria $(n=2,278)$. Thus, a total of 6,309 patients were included in the final analysis. The patient flowchart is shown in Supplementary Figure 1. Supplementary Table 1 illustrates the comparison of baseline characteristics between ineligible and eligible patients. Though statistically significant, differences in SBP, current smoking status, hypertension, and lipid parameters were not clinically relevant. In addition, the table shows no significant differences in sex, BMI, hs-CRP, or GRACE risk score. 


\section{Baseline Characteristics}

The baseline characteristics are displayed in Table 1. The patients were $75.2 \%$ male, with a mean (SD) age of $60.1(10.06)$ years old, and a mean (SD) BMI of $26.2(10.57) \mathrm{kg} / \mathrm{m}^{2}$. The rates of diabetes and dyslipidemia were $44.4 \%(2,803)$ and $74.7 \%$ $(4,710)$, respectively. A total of 5,488 (87\%) patients were presented with unstable angina. A total of 6,155 (97.6\%) patients received aspirin, and 6,200 (98.3\%) took a statin with/without ezetimibe (20.1\%). Left main artery lesions were observed in $16.8 \%$, multivessel lesions in $58.9 \%$, CTO lesion in $16.7 \%$, and lesions $>20 \mathrm{~mm}$ in $61 \%$ of patients. When we evaluated baseline characteristics according to median GRACE score, patients with a GRACE score $>91$ vs. 91 or less had higher BMI; had more cardiovascular risk factors including current smoking, hypertension, diabetes, low-density lipoprotein cholesterol levels, TC, and TG; and were less likely to be receiving statins. The rates of female and AMI were higher in the high-GRACE group. Besides, patients in the highGRACE group were likely to have a higher SYNTAX score and there was no statistically significant difference for other angiographic parameters.

\section{Relationships of Cardiovascular Events With Lp(a)}

The fully adjusted multivariable relationships between cardiovascular events and $\mathrm{Lp}(\mathrm{a})$ levels stratified according to GRACE score are shown in Table 2. A total of 310 (4.9\%) incident cardiovascular events occurred during 18 months of follow-up. Of the overall population, higher GRACE scores $(\geq 91$

TABLE 2 | Risk of cardiovascular events according to lipoprotein(a) and GRACE score.

\begin{tabular}{|c|c|c|c|}
\hline & No. (\%) & HR (95\% Cl) & $P$-value* \\
\hline \multicolumn{4}{|l|}{ LP(a) tertiles } \\
\hline $\mathrm{T} 1$ ( $\leq 7 \mathrm{mg} / \mathrm{dL})$ & $92(4.2)$ & 1 (reference) & 0.138 \\
\hline T2 (7-23 mg/dL) & $100(4.9)$ & $1.11(0.83-1.49)$ & 0.472 \\
\hline T3 (>23 mg/dL) & $118(5.8)$ & 1.33 (0.99-1.76) & 0.051 \\
\hline \multicolumn{4}{|l|}{ GRACE score } \\
\hline$\leq 91$ & $130(3.9)$ & 1 (reference) & - \\
\hline$>91$ & $180(6.1)$ & 1.50 (1.19-1.89) & 0.001 \\
\hline \multicolumn{4}{|l|}{ GRACE score $\leq 91$} \\
\hline \multicolumn{4}{|l|}{ LP(a) tertiles } \\
\hline $\mathrm{T} 1$ ( $\leq 7 \mathrm{mg} / \mathrm{dL})$ & $49(4)$ & 1 (reference) & 0.643 \\
\hline T2 (7-22 mg/dL) & $44(4.1)$ & $0.96(0.64-1.45)$ & 0.85 \\
\hline T3 (>22 mg/dL) & 37 (3.5) & $0.82(0.53-1.27)$ & 0.366 \\
\hline \multicolumn{4}{|l|}{ GRACE score>91 } \\
\hline \multicolumn{4}{|l|}{ LP(a) tertiles } \\
\hline $\mathrm{T} 1$ ( $\leq 7 \mathrm{mg} / \mathrm{dL})$ & $43(4.4)$ & 1 (reference) & 0.002 \\
\hline T2 (7-24 mg/dL) & $56(5.7)$ & $1.31(0.86-1.98)$ & 0.205 \\
\hline T3 (>24 mg/dL) & $81(8.2)$ & $1.94(1.32-2.84)$ & 0.001 \\
\hline
\end{tabular}

GRACE, Global Registry of Acute Coronary Events; Lp(a), lipoprotein(a). *Adjusted for age, sex, BMI, smoking status, hypertension, previous MI, previous stroke, SYNTAX score, number of stents, total length of stents, $h s-C R P, H D L-C, L D L-C$, lipid-lowering medication use. $P$ value for interaction $<0.001$. vs. $<91)$ were associated with an increased risk of cardiovascular events (HR: 1.50, 95\% CI: 1.19-1.89, $P<0.001$ ). However, no significant cardiovascular risk was associated with $\mathrm{Lp}$ (a) levels according to $\operatorname{Lp}(\mathrm{a})$ tertiles (tertile 2 vs. tertile 1: HR 1.11, 95\% CI: $0.83-1.49, P=0.472$; tertile 3 vs. tertile 1 : HR 1.33 , $95 \% \mathrm{CI}$ : $0.99-1.76, P=0.051)$. Nevertheless, when the overall population was stratified by GRACE score of 91 or less vs. $>91$ and tertiles of $\mathrm{Lp}$ (a) levels, a higher Lp(a) level was significantly associated with cardiovascular events only when the GRACE score was $>91$ (tertile 2 vs. tertile 1: HR 1.31, 95\% CI: $0.86-1.98, P=0.205$; tertile 3 vs. tertile 1: HR 1.94, 95\% CI: $1.32-2.84, P=0.001 ; P$ $=0.002$ ). Moreover, no such significant correlation between cardiovascular events and Lp(a) emerged in the case of GRACE score of 91 or less. We also found that there was a significant interaction for cardiovascular events between $\mathrm{Lp}(\mathrm{a})$ tertiles and dichotomized GRACE score $(P<0.001)$.

The cumulative incidence of cardiovascular events over time stratified by $\mathrm{Lp}$ (a) tertiles in the case of GRACE score 91 or less (Figure 1A) or $>91$ (Figure 1B) during an 18-months period is shown. In patients with GRACE score $>91$, increasing Lp(a) was associated with a greater cumulative incidence of cardiovascular events over time $(P<0.002)$, whereas patients with GRACE score of 91 or less were not $(P=0.706)$.

Sensitivity analyses are described in Table 3 by presenting the associations between cardiovascular events and $\mathrm{Lp}(\mathrm{a})$ levels stratified according to GRACE scores in patients with or without diabetes. Similar to the overall population, higher Lp(a) levels in patients with diabetes were significantly associated with cardiovascular events only when the GRACE score was $>92$ (tertile 2 vs. tertile 1: HR 1.30, 95\% CI: 0.73-2.31, $P=0.368$; tertile 3 vs. tertile $1:$ HR 1.93 , 95\% CI: $1.14-3.25$, $P=0.014 ; P=0.039$ ), but not when GRACE scores were 92 or less. However, no such relationship was found in patients without diabetes.

TABLE 3 | Risk of cardiovascular events according to lipoprotein(a) and GRACE score in patients with or without diabetes.

\begin{tabular}{|c|c|c|c|c|}
\hline & \multirow{2}{*}{$\begin{array}{c}\text { Diabetes } \\
\text { HR }(95 \% \mathrm{Cl})\end{array}$} & \multirow[b]{2}{*}{$P$-value* } & \multirow{2}{*}{$\frac{\text { Non-diabetes }}{\text { HR }(95 \% \mathrm{Cl})}$} & \multirow[b]{2}{*}{$P$-value* } \\
\hline & & & & \\
\hline \multicolumn{5}{|c|}{ GRACE score $\leq$ median } \\
\hline \multicolumn{5}{|c|}{ LP(a) tertiles } \\
\hline T1 & 1 (reference) & 0.308 & 1 (reference) & 0.875 \\
\hline T2 & $0.70(0.39-1.27)$ & 0.244 & $1.16(0.59-2.27)$ & 0.675 \\
\hline T3 & $1.12(0.65-1.92)$ & 0.68 & $1.18(0.60-2.34)$ & 0.633 \\
\hline \multicolumn{5}{|c|}{ GRACE score > median } \\
\hline \multicolumn{5}{|c|}{ LP(a) tertiles } \\
\hline T1 & 1 (reference) & 0.039 & 1 (reference) & 0.151 \\
\hline T2 & $1.30(0.73-2.31)$ & 0.368 & $1.24(0.71-2.18)$ & 0.45 \\
\hline T3 & $1.93(1.14-3.25)$ & 0.014 & $1.67(0.98-2.84)$ & 0.059 \\
\hline
\end{tabular}

GRACE, Global Registry of Acute Coronary Events; Lp(a), lipoprotein(a). *Adjusted for age, sex, BMI, smoking status, hypertension, previous MI, previous stroke, SYNTAX score, number of stents, total length of stents, hs-CRP, HDL-C, LDL-C, lipid-lowering medication use. 

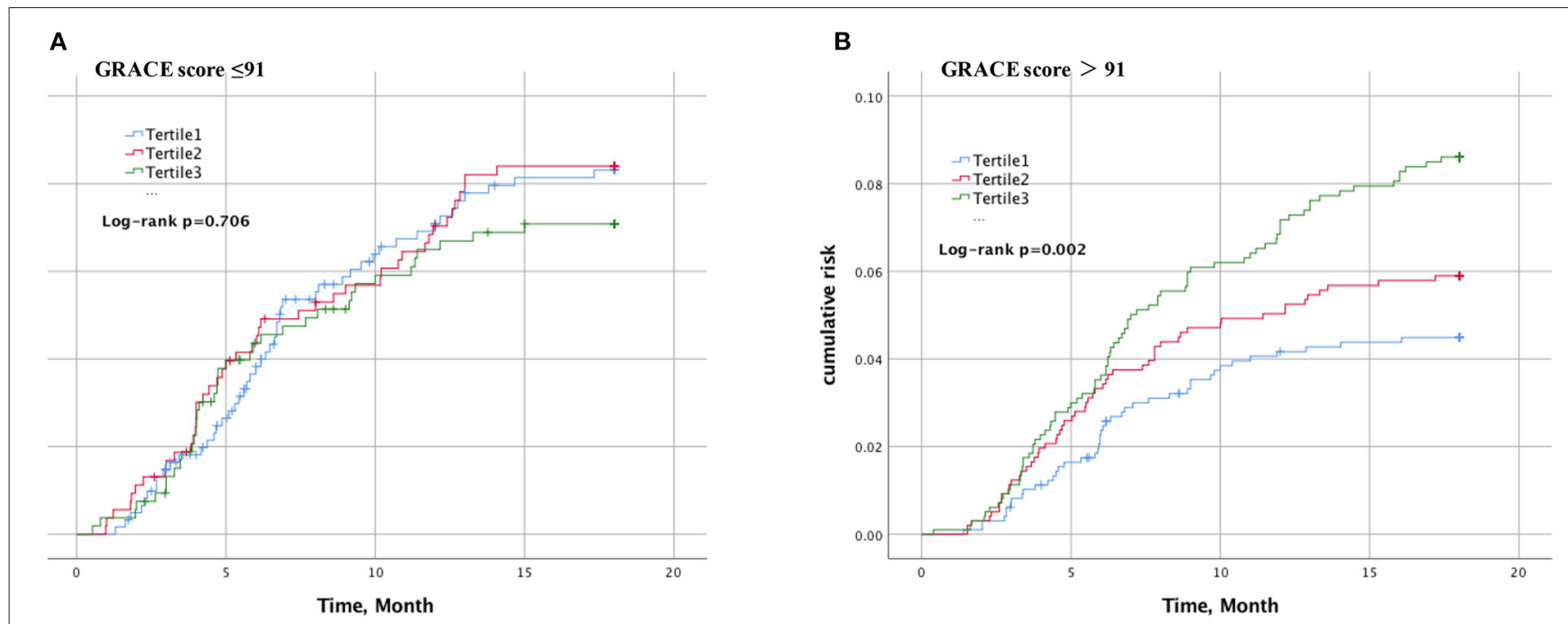

FIGURE 1 | Kaplan-Meier curve of cardiovascular events according to lipoprotein(a) and GRACE score. The cumulative incidence of cardiovascular events over time stratified by Lp(a) tertiles in the case of GRACE score 91 or less (A) or $>91$ (B).

\section{DISCUSSION}

\section{Main Findings}

Our findings demonstrated for the first time that there was a synergistic effect between GRACE score and on-statins Lp(a) levels on cardiovascular risk for ACS patients undergoing PCI. $\mathrm{Lp}(\mathrm{a})$-mediated cardiovascular risk only appears in case of greater risk of cardiovascular events (GRACE score > 91), but no such relationship exists in case of GRACE scores of 91 or less.

Studies have shown that $\mathrm{Lp}(\mathrm{a})$-lowering treatments might only be effective at high $L p(a)$ levels (20). However, the question is what the threshold is of $\operatorname{Lp}(\mathrm{a})$ for patients with a high cardiovascular risk. A meta-analysis including 7 randomized controlled trials (RCT) and 29069 statin-treated patients found that patients with $\mathrm{Lp}(\mathrm{a})>50 \mathrm{mg} / \mathrm{dL}$ were associated with a $40 \%$ higher cardiovascular risk (21). Further, Lp(a) levels $<50 \mathrm{mg} / \mathrm{dl}$ are recommended by the American Heart Association/American College of Cardiology cholesterol guidelines as optimal (5). However, as noted by the Copenhagen data (22) and randomized trials $(2,3)$, patients with $25-50 \mathrm{mg} / \mathrm{dl} \mathrm{Lp}$ (a) still carry a high risk of cardiovascular events, which was ignored by the recommendation. Actually, $\mathrm{Lp}(\mathrm{a})$ levels $<30 \mathrm{mg} / \mathrm{dl}$ might be deemed optimal and the risk could be almost negligible (8). For example, Erqou et al. (23) suggested that ASCVD risk starts to accrue from a $\mathrm{Lp}$ (a) level of $25-30 \mathrm{mg} / \mathrm{dL}$. The present analysis suggests that a significant cardiovascular risk appears with an $\mathrm{Lp}$ (a) level of $24 \mathrm{mg} / \mathrm{dL}$ or more in the case of a GRACE score of 91 or more, providing additional evidence. In addition, patients with $\mathrm{Lp}$ (a) levels higher than $25-30 \mathrm{mg} / \mathrm{dl}$ accounted for $30 \%$ of the global population, $\sim 2$ billion people with atherogenic properties 8 ).

Prior evidence in patients without previous CVD from epidemiological studies (23), Mendelian randomization studies (24), and genome-wide association studies $(25,26)$ have conclusively shown that $\mathrm{Lp}(\mathrm{a})$ is closely related to cardiovascular risk. A study included a total of 63,746 coronary artery disease (CAD) cases and 130,681 controls, indicating that the most potent genetic association with CAD was the LPA locus, which was more potent than LDL-, PCSK9-, and 9p21-related variants (26). Epidemiological studies $(24,27,28)$, genomewide association studies, and Mendelian randomization studies $(25,29)$ have shown that patients with high $\operatorname{Lp}(\mathrm{a})$ were likely to have a greater risk of death, myocardial infarction, and stroke and the associations were causal. A meta-analysis included 11 studies for a total of 18,978 subjects with established CAD and found that $\mathrm{Lp}(\mathrm{a})$ was significantly associated with cardiovascular risk (30). A pre-specified analysis of the placebo-controlled ODYSSEY Outcomes trial in 18,924 patients with recent ACS indicated that $\mathrm{Lp}(\mathrm{a})$ predicted the risk of MACE after recent ACS and suggested that $\mathrm{Lp}(\mathrm{a})$ was an independent predictor of cardiovascular risk and should be a treatment target for patients with ACS (31). A post hoc analysis of the FOURIER trial (Further Cardiovascular Outcomes Research with PCSK9 Inhibition in Subjects With Elevated Risk) suggested that an increased risk of venous thromboembolism (VTE) was significantly related to the increased Lp (a) but not LDL-C levels (32). Our findings further indicated that the relationship between $\mathrm{Lp}(\mathrm{a})$ and cardiovascular events could be affected by the GRACE score. Actually, a previous study demonstrated that the relationship was also mediated by hsCRP levels, and the association only existed in patients with hsCRP levels $\geq 2 \mathrm{mg} / \mathrm{L}$ but not in those with hsCRP $<2 \mathrm{mg} / \mathrm{L}$ (33).

However, the mechanisms responsible for the association between $\mathrm{Lp}(\mathrm{a})$ and cardiovascular risk remain uncertain. $\mathrm{Lp}(\mathrm{a})$ directly promotes the formation of atherosclerotic plaques as the same as LDL cholesterol because of the cholesterol component of $\mathrm{Lp}(\mathrm{a})$. Moreover, the structural homology of apo(a) with plasminogen can lead to pro-thrombosis/antifibrinolysis effects by interfering with endogenous fibrinolysis (22). In addition, apo(a) carries $85 \%$ of the pro-inflammatory 
oxidized phospholipids, which could damage the intima of coronary arteries and facilitate the rupture of plaques (34).

\section{Clinical Implication}

Based on our findings, these data suggest that patients with Lp(a)-mediated cardiovascular risk could be further identified by selecting patients with greater risk of cardiovascular events. Thus, it could allow us to more accurately identify people who might benefit the most from LP(a)-lowering treatment.

\section{Limitations}

This study had several limitations. First, although we adjusted for a wide range of confounders, residual confounding factors cannot be excluded in this cohort study. Second, the Lp(a) assay measured Lp(a) mass was less than ideal. Third, Lp(a) levels in this study were much lower than other studies in western countries. So the results might not be generalizable to other ethnic groups.

Fourth, it should also be noted that the proportion of patients with unstable angina in this study was much higher than in previous studies on ACS. Finally, the study was exploratory and the optimal cutoff value of the GRACE score and Lp(a) for identifying patients with high cardiovascular risk must be validated in RCTs.

\section{CONCLUSIONS}

In patients undergoing PCI for ACS, there was a synergistic effect on cardiovascular risk between GRACE score and on-statins $\mathrm{Lp}(\mathrm{a})$ levels. This finding could help us to more accurately identify patients who would benefit most from Lp(a)-lowering treatments. However, the findings should be repeated in more randomized, controlled trials.

\section{REFERENCES}

1. Cannon CP, Blazing MA, Giugliano RP, McCagg A, White JA, Theroux P, et al. Ezetimibe added to statin therapy after acute coronary syndromes. $N$ Engl J Med. (2015) 372:2387-97. doi: 10.1056/NEJMoa1410489

2. Khera AV, Everett BM, Caulfield MP, Hantash FM, Wohlgemuth J, Ridker PM, et al. Lipoprotein(a) concentrations, rosuvastatin therapy, and residual vascular risk: an analysis from the JUPITER Trial (Justification for the Use of Statins in Prevention: an Intervention Trial Evaluating Rosuvastatin). Circulation. (2014) 129:635-42. doi: 10.1161/CIRCULATIONAHA.113.004406

3. Albers JJ, Slee A, O’Brien KD, Robinson JG, Kashyap ML, Kwiterovich PO Jr, et al. Relationship of apolipoproteins A-1 and B, and lipoprotein(a) to cardiovascular outcomes: the AIM-HIGH trial (Atherothrombosis Intervention in Metabolic Syndrome with Low HDL/High Triglyceride and Impact on Global Health Outcomes). J Am Coll Cardiol. (2013) 62:15759. doi: 10.1016/j.jacc.2013.06.051

4. Nestel PJ, Barnes EH, Tonkin AM, Simes J, Fournier M, White $\mathrm{HD}$, et al. Plasma lipoprotein(a) concentration predicts future coronary and cardiovascular events in patients with stable coronary heart disease. Arterioscler Thromb Vasc Biol. (2013) 33:2902-8. doi: 10.1161/ATVBAHA.113.302479

5. Grundy SM, Stone NJ, Bailey AL, Beam C, Birtcher KK, Blumenthal RS, et al. AHA/ACC/AACVPR/AAPA/ABC/ACPM/ADA/AGS/APhA/

\section{DATA AVAILABILITY STATEMENT}

The original contributions presented in the study are included in the article/Supplementary Material, further inquiries can be directed to the corresponding author/s.

\section{ETHICS STATEMENT}

The studies involving human participants were reviewed and approved by the institutional review board of Beijing Anzhen Hospital. The ethics committee waived the requirement of written informed consent for participation.

\section{AUTHOR CONTRIBUTIONS}

$\mathrm{YZ}$, JZ, and $\mathrm{CH}$ contributed to the conception and design of this study. $\mathrm{CH}$ wrote this article. All authors made contribution to collect, analyze data, read, and approved the final manuscript.

\section{FUNDING}

This work was supported by a grant from the National Key Research and Development Program of China (2017YFC0908800), Beijing Municipal Administration of Hospitals' Ascent Plan (DFL20150601) and Mission plan (SML20180601), and Beijing Municipal Health Commission Project of Science and Technology Innovation Center (PXM2019_026272_000006)(PXM2019_026272_000005).

\section{SUPPLEMENTARY MATERIAL}

The Supplementary Material for this article can be found online at: https://www.frontiersin.org/articles/10.3389/fcvm. 2021.637366/full\#supplementary-material

ASPC/NLA/PCNA guideline on the management of blood cholesterol: a report of the american college of cardiology/american heart association task force on clinical practice guidelines. Circulation. (2019) 139:e1082-143. doi: 10.1161/CIR.0000000000000700

6. Task Force for the Management of Dyslipidaemias of the European Society of Cardiology (ESC) and European Atherosclerosis Society (EAS). ESC/EAS guidelines for the management of dyslipidaemias. Eur Heart J. (2016). 37:2999-3058. doi: 10.1093/eurheartj/ehw272

7. Tsimikas S, Fazio S, Ferdinand KC, Ginsberg HN, Koschinsky ML, Marcovina SM, et al. NHLBI Working Group recommendations to reduce lipoprotein(a)mediated risk of cardiovascular disease and aortic stenosis. J Am Coll Cardiol. (2018) 71:177-92. doi: 10.1016/j.jacc.2017.11.014

8. Tsimikas Sotirios. A test in context: lipoprotein(a): diagnosis, prognosis, controversies, and emerging therapies. J Am Coll Cardiol. (2017). 69:692711. doi: 10.1016/j.jacc.2016.11.042

9. Eagle KA, Lim MJ, Dabbous OH, Pieper KS, Goldberg RJ, Van de Werf F, et al. A validated prediction model for all forms of acute coronary syndrome: estimating the risk of 6-month postdischarge death in an international registry. JAMA. (2004) 291:2727-33. doi: 10.1001/jama.291.22.2727

10. Fox KAA, FitzGerald G, Puymirat E, Huang W, Carruthers K, Simon T. Should patients with acute coronary disease be stratified for management according to their risk? Derivation, external validation and outcomes using the updated GRACE risk score. Should patients with acute coronary disease be stratified for management according to their risk? Derivation, external 
validation and outcomes using the updated GRACE risk score. BMJ Open. (2014) 4:e004425. doi: 10.1136/bmjopen-2013-004425

11. Collet JP, Thiele H, Barbato E, Barthélémy O, Bauersachs J, Bhatt DL, et al. ESC Guidelines for the management of acute coronary syndromes in patients presenting without persistent ST-segment elevation. Eur Heart J. (2020) ehaa575. doi: 10.1093/eurheartj/ehaa575

12. Fox KA, Dabbous OH, Goldberg RJ, Pieper KS, Eagle KA, Van de Werf F, et al. Prediction of risk of death and myocardial infarction in the six months after presentation with acute coronary syndrome: prospective multinational observational study (GRACE). BMJ. (2006) 333:1091. doi: 10.1136/bmj.38985.646481.55

13. Neumann FJ, Sousa-Uva M, Ahlsson A, Alfonso F, Banning AP, Benedetto U, et al. ESC/EACTS Guidelines on myocardial revascularization. Eur Heart J. (2019) 40:87-165. doi: 10.1093/eurheartj/ehy394

14. Cutlip DE, Windecker S, Mehran R, Boam A, Cohen DJ, van Es GA, et al. Clinical end points in coronary stent trials: a case for standardized definitions. Circulation. (2007) 115:234451. doi: 10.1161/CIRCULATIONAHA.106.685313

15. Thygesen K, Alpert JS, Jaffe AS, Chaitman BR, Bax JJ, Morrow DA, et al. Fourth universal definition of myocardial infarction. Circulation. (2018) 138:e618-51. doi: 10.1161/CIR.0000000000000617

16. Hicks KA, Tcheng JE, Bozkurt B, Chaitman BR, Cutlip DE, Farb A, et al. ACC/AHA key data elements and definitions for cardiovascular endpoint events in clinical trials: a report of the american college of cardiology/american heart association task force on clinical data standards (Writing Committee to Develop Cardiovascular Endpoints Data Standards). Circulation. (2015). 132:302-61. doi: 10.1161/CIR.0000000000000156

17. Roffi M, Patrono C, Collet JP, Mueller C, Valgimigli M, Andreotti F, et al. ESC Guidelines for the management of acute coronary syndromes in patients presenting without persistent ST-segment elevation: Task Force for the Management of Acute Coronary Syndromes in Patients Presenting without Persistent ST-Segment Elevation of the European Society of Cardiology (ESC). Eur Heart J. (2016). 37:267-315. doi: 10.1093/eurheartj/ehv320

18. Cosentino F, Grant PJ, Aboyans V, Bailey CJ, Ceriello A, Delgado V, et al. (2019). ESC Guidelines on diabetes, pre-diabetes, cardiovascular diseases developed in collaboration with the EASD. Eur Heart J. (2020) 41:255323. doi: 10.1093/eurheartj/ehz486

19. Williams B, Mancia G, Spiering W, Agabiti Rosei E, Azizi M, Burnier M, et al. ESC/ESH Guidelines for the management of arterial hypertension. Eur Heart J. (2018) 39:3021-104. doi: 10.1093/eurheartj/ehy339

20. Najam O, Ray KK. Lp(a) and cardiovascular disease -Has the phoenix finally risen from the ashes?Eur Heart J. (2019) 40:2771-4. doi: 10.1093/eurheartj/ehz016

21. Willeit P, Ridker PM, Nestel PJ, Simes J, Tonkin AM, Pedersen TR, et al. Baseline and on-statin treatment lipoprotein(a) levels for prediction of cardiovascular events: individual patient-data meta-analysis of statin outcome trials. Lancet. (2018) 392:1311-20. doi: 10.1016/S0140-6736(18)31652-0

22. Nordestgaard BG, Chapman MJ, Ray K, Bore'n J, Andreotti F, Watts GF, et al. Lipoprotein(a) as a cardiovascular risk factor: current status. Eur Heart J. (2010) 31:2844-53. doi: 10.1093/eurheartj/ehq386

23. Emerging Risk Factors Collaboration, Erqou S, Kaptoge S, Perry PL, Di Angelantonio E, Thompson A, et al. Lipoprotein(a) concentration and the risk of coronary heart disease, stroke, and nonvascular mortality. JAMA. (2009) 302:412-23. doi: 10.1001/jama.2009.1063

24. Kamstrup PR, Tybjærg-Hansen A, Nordestgaard BG. Extreme lipoprotein(a) levels and improved cardiovascular risk prediction. J Am Coll Cardiol. (2013) 61:1146-56. doi: 10.1016/j.jacc.2012.12.023

25. Clarke R, Peden JF, Hopewell JC, Kyriakou T, Goel A, Heath SC, et al. Genetic variants associated with $\mathrm{Lp}$ (a) lipoprotein level and coronary disease. $\mathrm{N}$ Engl J Med. (2009) 361:2518-28. doi: 10.1056/NEJMoa0902604

26. CARDIoGRAMplusC4D Consortium, Deloukas P, Kanoni S, Willenborg C, Farrall M, Assimes TL, et al. Large-scale association analysis identifies new risk loci for coronary artery disease. Nat Genet. (2013) 45:2533. doi: $10.1038 /$ ng. 2480

27. Willeit P, Kiechl S, Kronenberg F, Witztum JL, Santer P, Mayr M, et al. Discrimination and net reclassification of cardiovascular risk with lipoprotein(a): prospective 15-year outcomes in the Bruneck Study. J Am Coll Cardiol. (2014) 64:851-60. doi: 10.1016/j.jacc.2014.03.061

28. Langsted A, Nordestgaard BG, Kamstrup PR. Elevated lipoprotein(a) and risk of ischemic stroke. J Am Coll Cardiol. (2019) 74:5466. doi: 10.1016/j.jacc.2019.03.524

29. Langsted A, Kamstrup PR, Nordestgaard BG. lipoprotein(a) and high risk of mortality. Eur Heart J. (2019) 40:2760-70. doi: 10.1093/eurheartj/ehy902

30. O'Donoghue ML, Morrow DA, Tsimikas S, Sloan S, Ren AF, Hoffman EB, et al. Lipoprotein(a) for risk assessment in patients with established coronary artery disease. J Am Coll Cardiol. (2014) 63:520-7. doi: 10.1016/j.jacc.2013.09.042

31. Bittner VA, Szarek M, Aylward PE, Bhatt DL, Diaz R, Edelberg JM, et al. Effect of alirocumab on lipoprotein(a) and cardiovascular risk after acute coronary syndrome. J Am Coll Cardiol. (2020) 75:13344. doi: 10.1016/j.jacc.2019.10.057

32. Marston NA, Gurmu Y, Melloni GEM, Bonaca M, Gencer B, Sever PS, et al. The Effect of PCSK9 (Proprotein convertase subtilisin/kexin type 9) inhibition on the risk of venous thromboembolism. Circulation. (2020) 141:1600-7. doi: 10.1161/CIRCULATIONAHA.120.046397

33. Puri R, Nissen SE, Arsenault BJ, St John J, Riesmeyer JS, Ruotolo G, et al. Effect of C-reactive protein on lipoprotein(a)-associated cardiovascular risk in optimally treated patients with high-risk vascular disease: a prespecified secondary analysis of the ACCELERATE trial. JAMA Cardiol. (2020) 5:18. doi: 10.1001/jamacardio.2020.2413

34. Nordestgaard BG, Langsted A. Lipoprotein(a) as a cause of cardiovascular disease: insights from epidemiology, genetic and biology. J Lipid Res. (2016) 57:1953-75. doi: 10.1194/jlr.R071233

Conflict of Interest: The authors declare that the research was conducted in the absence of any commercial or financial relationships that could be construed as a potential conflict of interest.

Copyright $\odot 2021 \mathrm{Hu}$, Liu, Han, Sun, Cheng, Liu, Gao, Zhou, Zhang and Zhao. This is an open-access article distributed under the terms of the Creative Commons Attribution License (CC BY). The use, distribution or reproduction in other forums is permitted, provided the original author(s) and the copyright owner(s) are credited and that the original publication in this journal is cited, in accordance with accepted academic practice. No use, distribution or reproduction is permitted which does not comply with these terms. 